\title{
Socio-sexuality and episodic memory function in women: further evidence of an adaptive "mating mode"
}

\author{
David S. Smith • Benedict C. Jones • Kevin Allan
}

Published online: 7 February 2013

(C) Psychonomic Society, Inc. 2013

\begin{abstract}
The functionalist memory perspective predicts that information of adaptive value may trigger specific processing modes. It was recently demonstrated that women's memory is sensitive to cues of male sexual dimorphism (i.e., masculinity) that convey information of adaptive value for mate choice because they signal health and genetic quality, as well as personality traits important in relationship contexts. Here, we show that individual differences in women's mating strategies predict the effect of facial masculinity cues upon memory, strengthening the case for functional design within memory. Using the revised socio-sexual orientation inventory, Experiment $1 \mathrm{dem}-$ onstrates that women pursuing a short-term, uncommitted mating strategy have enhanced source memory for men with exaggerated versus reduced masculine facial features, an effect that reverses in women who favor long-term committed relationships. The reversal in the direction of the effect indicates that it does not reflect the sex typicality of male faces per se. The same pattern occurred within women's source memory for women's faces, implying that the memory bias does not reflect the perceived attractiveness of faces per se. In Experiment 2, we reran the experiment using men's faces to establish the reliability of the core finding and replicated Experiment 1's results. Masculinity cues may therefore trigger a specific mode within women's episodic memory. We discuss why this mode may be triggered by female faces and its possible role in mate choice. In so doing, we draw upon the encoding specificity principle and the idea that episodic memory limits the scope of stereotypical inferences about male behavior.
\end{abstract}

\footnotetext{
D. S. Smith $\cdot$ K. Allan $(\bowtie)$

School of Psychology, College of Life Sciences and Medicine, University of Aberdeen, AB24 2UB Aberdeen, UK

e-mail:k.allan@abdn.ac.uk

B. C. Jones

Institute of Neuroscience and Psychology, University of Glasgow, G12 8QB Glasgow, UK
}

Keywords Adaptive memory $\cdot$ Episodic memory $\cdot$ Mate choice $\cdot$ Faces $\cdot$ Sexual dimorphism

\section{Introduction}

Nairne and colleagues have argued that the functional organization of memory is likely to have been influenced by natural selection and that the consequences of specific selection pressures might emerge if we examine memory for information of adaptive value (Nairne, Pandierada, \& Thompson, 2008; see also Sherry \& Schacter, 1987). For example, recall is significantly enhanced when information is encoded in relation to its value for survival in an ancestral grasslands context (e.g., Burns, Burns \& Hwang, 2011; Nairne, Thompson, \& Pandeirada, 2007). Other memory biases of potential importance from this functionalist perspective have been discovered, including calorie biases in spatial memory for food (New, Krasnow, Truxaw, \& Gaulin, 2007) and trade-offs that regulate the social exchange of knowledge about the past (Allan, Midjord, Martin, \& Gabbert, 2012; Jaeger, Lauris, Selmeczy, \& Dobbins, 2012). In contrast, memory biases triggered by members of the opposite sex that might affect an individual's reproductive fitness have proven to be surprisingly elusive (see, e.g., Anderson et al., 2010).

In two recent papers (Allan, Jones, DeBruine, \& Smith, 2012; Smith, Jones, Feinberg, \& Allan, 2012), however, we used an ecological model of mate choice (Bateson \& Healy, 2005) to develop novel hypotheses about memory function in relation to a woman's reproductive fitness. We chose this approach because ecologists interested in the cognition that underlies mate choice have already laid some of the groundwork for a functionalist perspective by identifying circumstances where memory could play a crucial role (Bateson \& Healy, 2005). Bateson and Healy noted that one potentially serious problem could occur when mate choice is based 
upon perceptual cues that have conflicting connotations about a male's qualities (e.g., when a perceptual cue advertises both positive and negative characteristics in a potential mate). In such situations, memory could provide information from one's past experience that would help to resolve these ambiguities, potentially helping to optimize mate choice decisions (Bateson \& Healy, 2005).

In men, sexually dimorphic physical cues (see Fig. 1) may have precisely the double-edged quality highlighted by Bateson and Healy (2005). Moreover these cues systematically influence women's judgments about men's attractiveness as a partner (e.g., Perrett et al., 1998). Masculine characteristics in men are positively correlated with indices of good longterm health, such as low frequency and severity of past health problems (Rhodes, Chan, Zebrowitz, \& Simmons, 2006; Thornhill \& Gangestad, 2006) and other biomarkers of good physical condition (Gangestad, Thornhill, \& Garver-Apgar, 2010; Little et al., 2008; although see Penton-Voak, 2011). Moreover, testosterone levels in men are positively correlated with direct measures of immune system function (Rantala et al., 2012). Masculine characteristics in men are also positively correlated with measures of their dominance, such as social status (Mueller \& Mazur, 1996), physical strength (Fink, Neave, \& Seydel, 2007), and, potentially, fighting ability (Sell et al., 2009).

As compared with relatively feminine men, however, masculine men have an increased propensity to cheat on relationship partners (Hughes, Dispenza, \& Gallup, 2004),
Fig. 1 The effect of the Psychomorph transform (see the Method section for details) applied to an individual's facial image, in order to alter facial shape cues that signal sexual dimorphism. Top panel: Male exaggerated (i.e., masculinized) on the left, male reduced (i.e., feminized) on the right. Bottom panel: Female exaggerated (i.e., feminized) on the left, female reduced (i.e., masculinized) on the right
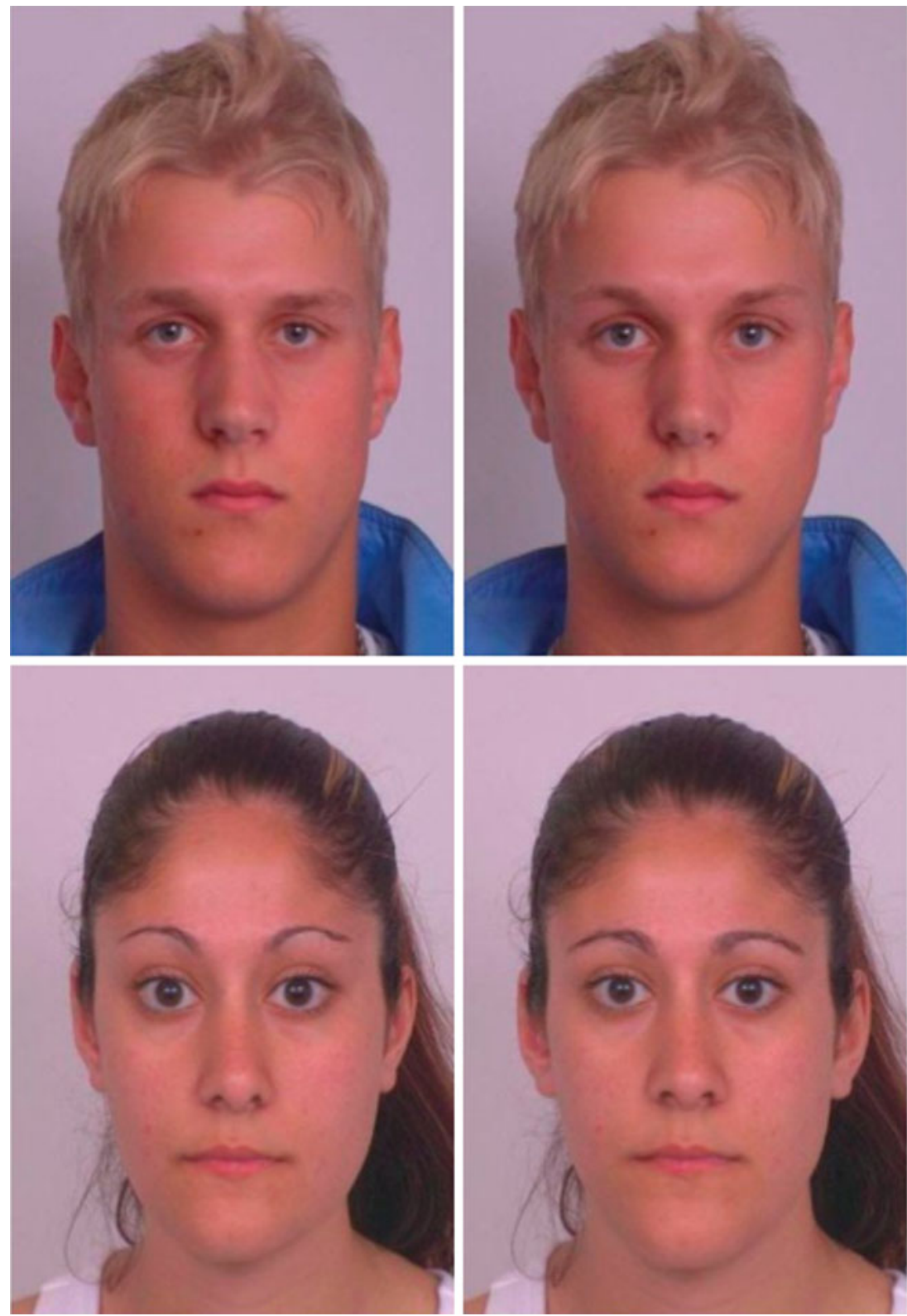
are more open to short-term uncommitted relationships (Boothroyd, Jones, Burt, DeBruine, \& Perrett, 2008), and are less likely to share resources fairly (Price, Kang, Dunn, \& Hopkins, 2011). As compared with relatively feminine men, masculine men are also perceived as being colder, more dishonest, and less likely to invest time in their offspring (e.g., Perrett et al., 1998). In choosing a partner, it has therefore been argued that women may trade off the advantages and disadvantages of relatively masculine or relatively feminine men by strategically adjusting the characteristics they desire according to different mating contexts (Fink \& Penton-Voak, 2002; Gangestad \& Simpson, 2000). This strategic trade-off hypothesis has generated a substantial and generally supportive literature that has revealed various forms of evidence for adaptive design within women's preferences for sexually dimorphic traits in men (recently reviewed in Little, Jones, \& DeBruine, 2011).

On the basis of this prior work, we suggested (Allan et al., 2012a; see also Smith et al., 2012) that women's evaluation of men in terms of their sexually dimorphic cues provides an ideal test of Bateson and Healy's (2005) ideas about the role of memory in mate choice. To reiterate, Bateson and Healy argued that memory can provide information that aids evaluation of men as potential partners, in situations where their physical characteristics have both positive and negative connotations. We argued that mate choice decisions based on apparent cues of sexual dimorphism invoke what is essentially stereotypical knowledge about the behavior of relatively masculine or feminine men within different relationship contexts. To decrease the risks associated with this gamble upon the veracity of one's stereotypes, we hypothesized that information associated with men who display desirable sexually dimorphic features may limit the scope of one's stereotypical views about such individuals. ${ }^{1}$ On the basis of this hypothesis, we made the following three novel predictions (Allan et al., 2012a). First, women should show an enhanced ability to retain information from encounters with men who display desirable versus less desirable sexually dimorphic characteristics. Second, this bias should be triggered specifically by cues of sexual dimorphism found to be attractive in men, and not by cues of sexual dimorphism found to be attractive in women. In relation to this prediction, it is worth reemphasizing that the attractiveness of the former (i.e., men's cues of sexual dimorphism) shows considerable, systematic, individual differences, while the attractiveness of the latter (i.e., women's cues of sexual dimorphism) is much more consistent. That is, women (and men) tend to rate feminized (sex-typical) features in women as more attractive than masculinized features (e.g., Perrett et al., 1998; Rhodes et al., 2006).

\footnotetext{
${ }^{1}$ The same point was made about episodic memory function more generally by Klein, Cosmides, Tooby, and Chance (2002).
}

Third, we made the additional assumption that men's mate choice is more dependent upon physical cues of attractiveness in women (see, e.g., Landolt, Lalumiere, \& Quinsey, 1995), and hence we predicted that men should show no analogous pattern of memory bias.

To test all three predictions, we examined whether preferences for sexually dimorphic features in the opposite sex and in the same sex predict memory for object images that are shown alongside facial images transformed to either enhance or reduce their sexually dimorphic structural features (Allan et al., 2012a; see also Tiddeman, Perrett, \& Burt, 2001, for image transform methods). We observed that women who currently preferred relatively masculine features in men's faces had enhanced two-alternative forced choice recognition of objects paired at encoding with masculinized versus feminized men. In contrast, women who currently preferred men with relatively feminine facial features showed the opposite pattern-that is, an enhanced ability to recognize studied objects previously viewed alongside feminized versus masculinized facial images of men. As was predicted, this memory bias was not present for objects paired at encoding with other women's faces, even though, as a group, our female participants found feminized versus masculinized women's faces significantly more attractive. Also as was predicted, the analogous bias was absent in male participants, even though they significantly preferred feminized versus masculinized women's faces. Overall, these findings revealed a novel pattern of sex differences in memory function, supporting our hypothesis that information linked to desirable men is valuable for women's mate choice (see also Smith et al., 2012) and that mere preference for a facial feature is not sufficient to drive the memory bias. It is also worth noting that these findings are somewhat counterintuitive, on the assumption that attractive faces of the opposite sex might be attention grabbing, for which there is behavioral evidence (e.g., Anderson et al., 2010). However, we did not observe a pattern of impaired memory for object details "peripheral" to preferred versus nonpreferred faces, and so the predictions of this attentional account do not appear to be borne out empirically.

The aim of the present article is to further explore the hypothesis that an adaptive mating mode exists within women's episodic memory. The hypothesis assumes that episodic details from encounters with men who display desirable sexually dimorphic features are functional for the particular mating strategy that a woman pursues in order to trade off the pros and cons associated with a relatively masculine or feminine partner. Hence, the hypothesis predicts that women who pursue mating strategies that select for specific sexually dimorphic characteristics in a partner should have enhanced memory for encounters with men who meet this specific criterion. A powerful way to test this prediction is to examine whether opposite patterns of memory bias occur in women whose mating strategies select for either relatively masculine or relatively feminine men. 
Preferences for relatively masculine men are heightened in women who favor short-term uncommitted relationships, and conversely, preferences for relatively feminine men are heightened in women who favor long-term committed relationships (Provost, Kormos, Kosakoski, \& Quinsey, 2006; Waynforth, Delwadia, \& Camm, 2005; although see Glassenberg, Feinberg, Jones, Little, \& DeBruine, 2010). Therefore, women who are more open to short-term, uncommitted relationships should show biases toward information associated with partners appropriate to that strategy - that is, relatively masculine men-whereas women who have a more restricted mating strategy that favors long-term committed relationships should show the opposite bias, toward information associated with relatively feminine men.

The primary goal of the two experiments reported here was to test these new predictions about the link between adaptive mating strategies and biases in women's memory function. A secondary goal was to gain further insight into the nature of the memory processes in women that are affected by cues of sexual dimorphism in men. Following recent work on adaptive memory (e.g., Bell, Buchner, Kroneisen, \& Giang, 2012; Nairne VanArsdall, Pandeirada, \& Blunt, 2012), we employed a source memory test (e.g., Johnson, Hashtroudi, \& Lindsay, 1993 ) that requires participants to recollect each individual encoding episode. Participants viewed a series of male and female facial images with either heightened or reduced sexual dimorphism, with each image enclosed by a green or yellow frame. Following a brief study-test interval, we re-presented each facial image and asked participants to recall the frame color. Following the source memory test, participants completed the revised socio-sexual orientation inventory (SOI-R; Penke \& Asendorpf, 2008a, b), a questionnaire that allows us to quantify their orientation toward restricted (i.e., long-term, committed) versus unrestricted (i.e., short-term, uncommitted) relationships.

Our hypothesis in Experiment 1 was that women with high SOI-R scores, indicating a relatively unrestricted socio-sexual orientation, should have better source memory for encounters with masculine versus feminine men. In contrast, women with low SOI-R scores, indicating a relatively restricted sociosexual orientation, should have better source memory for encounters with feminine versus masculine men.

\section{Experiment 1}

Method

\section{Participants}

Ninety heterosexual women (mean age $=23.3$ years; range, 18-43.6 years; $S D=5.77$ years), none of whom were currently taking hormonal contraceptives, participated.
Data were gathered using an online interface (for validation studies, see, e.g., Jones et al., 2007) approved by the Aberdeen School of Psychology ethics committee.

\section{Materials}

Psychomorph computer graphic software was employed to systematically manipulate structural features that convey sexual dimorphism in two-dimensional facial images (see Fig. 1; Tiddeman et al., 2001). Psychomorph ensures that only sexually dimorphic shape cues are altered, rather than color and texture cues. Forty two-dimensional, full-face, color images of young healthy male and female individuals were employed (20 males and 20 females). Transformations were based upon a total of 174 feature points delineated in each base image, from which the average male and female face shapes were first calculated. Using the linear facial difference between each feature point in the average male and female faces, masculinized faces were constructed by shifting each point in the base image halfway along the vector toward the position of the corresponding point on the average male face, while feminized faces involved shifting each point halfway along the vector toward the position of the corresponding point on the average female face shape. This method produces face images that differ systematically and reliably in their perceived masculinity and femininity (see DeBruine et al., 2006).

The 5-point Likert scale SOI-R (Penke \& Asendorpf, 2008b) was used to assess individual differences in participants' willingness to engage in uncommitted short-term sexual relationships versus committed long-term relationships. The SOI-R assesses participants' sexual behaviors (i.e., personal sexual histories), attitudes (i.e., dispositions toward uncommitted relationships), and desires (i.e., motivational states). A composite (i.e., average) score reflecting each participant's socio-sexual orientation can then be formed and used as a single index reflecting the degree to which the participant pursues a committed restricted (lower composite score) versus unrestricted uncommitted (higher composite score) mating strategy. Note that in their paper, Penke and Asendorpf (2008a) reported that a three-factor structure provided the best fit to the SOI-R data, using confirmatory factor analyses, loading onto different subsets of the SOI-R questions and respecting the distinction between separable attitude, desire, and behavior components. However, we find that it is only the composite (average) SOI-R score that predicts the effect of masculinity cues upon recollection, and not the subscale scores in isolation. For this reason, we focus below on the relationship between average SOI-R score and recollection.

During the encoding phase, participants viewed 20 male faces (10 exaggerated, 10 reduced in masculinity) and 20 female faces (10 exaggerated, 10 reduced in femininity). 
Each face was presented for $2 \mathrm{~s}$ centrally within a either a green or a yellow framing rectangle, with color randomly allocated to each face ensuring that each color occurred equally often in each $2 \times 2$ factorial condition of face sex and level of sexual dimorphism. During the memory phase, each participant viewed all 40 studied male and female faces again, with each face shown at the center of the screen above a green and a yellow rectangle positioned on the left and the right sides of the screen. Using their computer mouse, participants had to click within one of the rectangles to indicate the color of the frame associated with each face at study. The test was self-paced.

\section{Procedure}

Participants were shown onscreen instructions informing them that they would view a series of faces surrounded by a colored border and that they should simply pay attention to each face in turn. During the encoding phase, the presentation sequence of the 40 faces was randomized to eliminate order effects. Each face was displayed for $2 \mathrm{~s}$ with its accompanying colored frame (green or yellow). The color of frame that was associated with each face was randomly selected from one of four different orders, with five instances for each of the two colors being presented across the four different face conditions. After participants had seen all 40 faces, the sequence was repeated once again.

After a brief pause, participants were told that they would view each face again in a different randomized order. Their task was to recollect the frame color for each face, and to respond by clicking on either the green or the yellow rectangle that would be shown below each face. Once all 40 responses had been collected, the SOI-R questionnaire was completed, and then participants were thanked and debriefed.

\section{Results and discussion}

We begin by describing source memory performance as a function of the main independent variables. Group performance on the source memory task hovered at or just above the $50 \%$ chance level (male exaggerated, mean $=49.1 \%$, $S D=14.1$; male reduced, mean $=51.1 \%, S D=15.6$; female exaggerated, mean $=51.5 \%, S D=15.5$; female reduced, mean $=55.3 \%, S D=14.5)$. As these figures indicate, on average, our participants' memory tended to be better for episodes involving other women's faces per se than for men's faces. This was confirmed by an ANOVA (withinsubjects factors: face sex [male/female] and face shape [masculinized/feminized]), which produced only a significant main effect of gender, $F(1,89)=4.16, p<.05$. This finding replicates prior work demonstrating within-sex biases in women's memory for other women, which, in some papers, have been increased for attractive versus unattractive women's faces (e.g., Becker, Kenrick, Guerin, \& Maner, 2005; Maner et al., 2003; see also Rehnman \& Herlitz, 2007).

The results of this initial ANOVA confirm that at the group level, the manipulation of sexual dimorphism in men's and women's faces did not appear to produce any modulatory effect upon women's source memory. Although the face sex effect demonstrates that performance is not fully at floor across all conditions, it is possible that the absence of sexual dimorphism effects within each gender could be due to a floor effect. While we fully acknowledge this possibility, the absence of sexual dimorphism effects at the level of the group is consistent with our earlier findings (Allan et al., 2012b). That is, the enhancing effect of sexually dimorphic cues in men upon women's memory should systematically vary according to a woman's mating strategy. In fact, depending on that strategy, the direction of the effect should reverse. Some women should show enhanced memory for masculinized versus feminized men, while other women should show the reverse pattern. Hence, across a group of women, without taking into account mating strategies, we would expect that both effects would tend to cancel one another out. In terms of our experimental hypothesis, however, measures of this individual variability should predict the effect of men's sexual dimorphism upon women's memory.

Therefore, we specifically predict opposite effects of the face sexual dimorphism manipulation upon recollection depending on whether an individual scores high or low on the SOI-R. Although there are different statistical approaches that could be employed to test this hypothesis, to take full advantage of the continuous nature of the SOI-R variable, we employed an ANCOVA (see MacCallum, Zhang, Preacher, \& Rucker, 2007, for a discussion of various problems, including power issues, associated with dichotomizing continuous quantitative variables). The ANCOVA allowed us to determine whether individual differences in participants' average SOI-R scores predict systematic modulatory effects of men's cues to sexual dimorphism upon women's recollection, and this was indeed the case.

In our participants, mean SOI-R composite (i.e., average) score was $2.38(S D=0.79)$, and internal reliability was high (Cronbach's alpha $=.765)$. Note also that our participants' SOI-R data appear to fit with the norms provided by Penke (see http://www.larspenke.eu/en/research/soi-r.html) for heterosexual women 18-45 years of age. The dependent variable used in the ANCOVA was the percentage of correct responses on the source memory test. The ANCOVA (between-subjects factor, SOI-R; within-subjects factors, face sex [male/female] and face shape [masculinized/feminized]) produced a significant main effect of face shape, $F(1,88)=4.28, p<.05$, qualified by a significant two-way interaction between the covariate SOI-R and face shape, $F(1,88)=5.63, p=.02$, 
but the three-way interaction between SOI-R, face sex, and face shape was not significant, $F(1,88)=0.01, p=.91$.

To interpret the two-way interaction between SOI-R and face shape, which reflects a consistent effect of masculinized face shape within the male and female faces, we examined the correlation between SOI-R score and the average effect of the face shape manipulation on recollection of male and female faces. For these analyses, we calculated the difference between the percentage of correct responses on the source memory test for the masculine male face versus feminine male face conditions and, separately, for the masculine female face versus feminine female face conditions, and then we averaged the two difference scores together (i.e., we simply reconstructed the means involved in the two-way interaction). The correlation between SOI-R score and the average effect of face shape on source memory was significant and positive, $r(90)=.25, p=.02$. Figure 2 reveals the nature of the relationship captured within this correlation between SOI-R and the effect of masculinity cues upon women's memory; women with higher SOI-R scores were better able to recollect episodes where they viewed images of masculinized versus feminized faces, while participants with lower SOI-R scores were better able to recollect episodes where they viewed images of faces with feminized versus masculinized shape cues. As a final point, to further explore the two-way interaction in the ANCOVA, we also used a median split to form two participant groups, one high and one low in SOI-R. But, likely because of the reduction in statistical power (MacCallum et

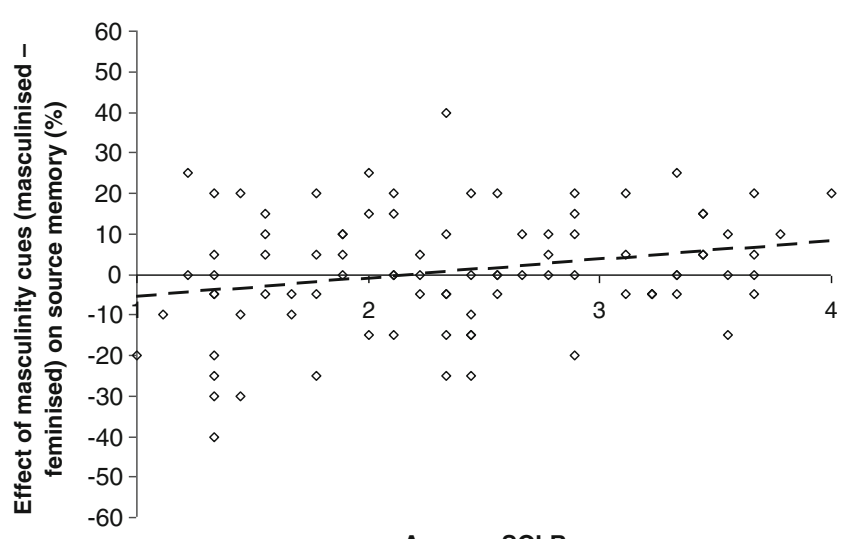

Average SOI-R

Fig. 2 Experiment 1 data showing the relationship between average SOI-R score and the effect of masculinizing versus feminizing faces upon source memory. The $y$-axis shows the difference in source memory for masculinized minus feminized faces, averaged across male and female faces (see the Results section). Higher average SOI-R scores (revealing a preference for short-term, uncommitted relationships) are associated with better source memory for masculinized versus feminized faces. In contrast, lower average SOI-R scores (revealing a preference for long-term, committed relationships) are associated with better source memory for feminized versus masculinized faces, $r(90)=$ $.25, p=.02$ al., 2007), we did not find evidence from this approach that sexual dimorphism produced significant patterns of memory enhancement in different directions in both groups.

Prior studies examining memory for attractive versus unattractive faces have found little evidence that male attractiveness enhances women's memory, even when individual-difference measures of potential importance for mating have been taken into account, such as menstrual cycle phase (Anderson et al., 2010) and the earlier, nonrevised version of the SOI (Maner et al., 2003). Instead, these prior studies suggest that women's memory is enhanced for other women (vs. men) and is also sensitive to the attractiveness of other women (see also Rehnman \& Herlitz, 2007). To allow comparison of these prior findings with our present data, we initially carried out an analysis that did not take into account the individual differences in SOI$\mathrm{R}$ scores that were of primary importance for our experimental hypothesis. The results of this initial analysis showed that women did in fact have enhanced ability to recollect the source detail (border color around facial images) associated with women's versus men's faces. But in addition, our data also suggest that source memory, overall, is better for masculinized than for feminized women's faces, which appears to go against earlier findings on the mnemonic effect of women's attractiveness (e.g., Maner et al., 2003).

When we examined how women's recollection covaried with their socio-sexual orientation, however, we observed a systematic modulatory effect of facial masculinity cues upon women's ability to recollect source detail from encounters with men and with women (cf. Maner et al., 2003). The pattern was essentially as we had predicted. That is, as women's socio-sexual orientation becomes less restricted (i.e., individuals become more open to short-term, uncommitted relationships), recollection is relatively enhanced by exaggerated cues of masculinity. As women's socio-sexual orientation becomes more restricted, however, the pattern reverses, and recollection becomes enhanced for faces displaying reduced versus enhanced cues of masculinity (see Fig. 2). This pattern replicates and extends findings from our prior study using women's preferences for masculinity or femininity in men's faces as a covariate of interest to predict recognition of objects that co-occurred alongside images of male faces. These prior findings strongly suggested that the memory bias was not due to the sex typicality of men's facial structure per se, because of the reversal in the apparent direction of the bias across individuals. A similar reversal occurs in the present data, confirming that the mnemonic effect of masculinity cues is not a simple enhancement triggered by male sex typicality.

In one respect, however, our findings differ from the previous findings upon which our predictions were based. Here, we observed that facial masculinity cues modulated women's source memory for both male and female faces, whereas in our 
prior studies, we observed effects on women's object recognition that were specific to cues of sexual dimorphism in male faces (Allan et al., 2012a) and male voices (Smith et al., 2012). This difference between the present and previous findings is important to acknowledge, because it appears to be at odds with the functionalist interpretation that mate choice is the driving factor that triggers the mnemonic effects of masculinity cues. One might argue that, on this interpretation, memory should be sensitive to cues of masculinity in men but insensitive to such cues in other women. However, we would question the premise that the mate choice hypothesis strictly requires a selective response from memory that is attuned to signals of sexual dimorphism in men but not women. It is quite feasible, instead, that the presence of the effect in women's faces could be a functionless by-product of a memory bias sensitive to cues of masculinity in men. By-products are characteristics that do not appear to contribute toward solving adaptive problems, showing no sign of functional design (see, e.g., Buss, Haselton, Shackelford, Bleske, \& Wakefield, 1998). However, they may remain as a feature if they incur little or no cost or if they are inextricably linked with another feature or trait that does confer some adaptive benefit (for more discussion, see Andrews, Gangestad, \& Mathews, 2002; Justin, 2007). We return to this point in the General Discussion section below.

Our present findings do, however, suggest that the memory bias is not driven by the perceived attractiveness of faces per se. As was noted in the introduction, while women's preferences for cues of masculinity in men's faces shows systematic individual differences, captured for example by the SOI-R, women's preferences for sexually dimorphic features in other women tends to be more consistent; women (and men) typically rate feminized versus masculinized facial features as more attractive in women (e.g., Perrett et al., 1998; Rhodes et al., 2006). Hence, our present finding, that higher SOI-R scores are related to enhanced memory for masculinized versus feminized women's faces, is contrary to a prediction based simply on perceived attractiveness that would apply generally to both men's and women's faces (see Allan et al., 2012a, for further evidence against this attractiveness interpretation).

In sum, our present findings extend the findings of three prior studies from our lab, one employing face stimuli (Allan et al., 2012b) and two employing voice stimuli (Smith et al., 2012). Nevertheless, because the present findings are novel, it would seem prudent to verify that they can be replicated, and so this was the aim of Experiment 2. We reran the procedure in an independent group of women participants, focusing on the recollection modulating effects of sexually dimorphic cues in men's faces. To reiterate, we expected to find that participants' socio-sexual orientation would predict the effect of facial masculinity cues upon women's ability to recollect and that the relationship would replicate the pattern observed in Experiment 1.

\section{Experiment 2}

Method

\section{Participants}

Seventy-one heterosexual women (mean age $=24.8$ years; range, $18.2-42.5$ years; $S D=6.01$ years), none of whom were currently taking hormonal contraceptives, participated. Data were gathered via the same online interface as that utilized in Experiment 1.

\section{Materials}

The stimuli were identical to those used in Experiment 1, except that we employed only male, and not female, faces. During encoding, participants viewed 20 male faces (10 exaggerated, 10 reduced in masculinity). During the memory phase, each participant viewed all 20 studied male faces again.

\section{Procedure}

With the exception of having no female faces, Experiment 2 was procedurally identical to Experiment 1 . All counterbalancing and randomizing factors, with regard to the order of masculinized and feminized faces and their corresponding colored frames, were as per Experiment 1.

\section{Results}

As was the case in Experiment 1, group performance on the source memory task hovered at or just above the $50 \%$ chance level (male exaggerated, $M=54.4 \%, S D=181$; male reduced, $M=47.3 \%, S D=17.7)$. Here, though, women's recollection of the border color around men's faces was significantly better for faces displaying exaggerated versus reduced cues of sexual dimorphism, $t(70)=2.62, p=.01$. The central issue for Experiment 2 was whether we could replicate the key finding from Experiment 1 - that is, the finding that opposite effects of sexual dimorphism occur depending on participants' SOI-R score. To test this hypothesis, once again we employed an ANCOVA to determine whether individual differences in participants' average SOI-R scores predicted systematic modulatory effects of men's sexual dimorphism upon women's recollection. The dependent variable once again was the percentage of correct responses on the source memory test. In these participants, mean SOI-R score was $2.24(S D=0.69)$, and internal reliability was high (Cronbach's alpha $=.76$ ).

The ANCOVA (between-subjects factor, SOI-R; withinsubjects factor, male face shape [masculinized/feminized]) produced a significant two-way interaction between face shape and SOI-R score, $F(1,69)=4.76, p=.033$. As was the case in Experiment 1, to elucidate the interaction, we 
examined the correlation between SOI-R score and the effect of male face shape on source memory. As was also observed in Experiment 1, the correlation was both significant and positive, $r(71)=.25, p<.05$. As is evident from Fig. 3 (and compare it with Fig. 2), women with less restricted socio-sexuality-that is, higher SOI-R scoresonce again showed enhanced ability to recollect encountering a masculinized versus feminized face, while women with more restricted socio-sexuality showed the opposite pattern. As was also the case in Experiment 1, we examined the data using a median split to form two separate groups, one high and one low in SOI. But using this dichotomizing method, we were unable to detect any evidence of significant sexual dimorphism effects in either group.

In sum, these findings from Experiment 2 show that overall, women's memory can benefit from the presence of exaggerated versus reduced masculinity cues in men's faces (see Smith et al., 2012, for a similar finding using cues of vocal masculinity). However, the central point is that the findings from Experiment 2 replicate a key result from Experiment 1. That is, and as was predicted, women's ability to recollect encounters with men is modulated by cues of sexual dimorphism that signal the suitability of a man for the mating strategy (short-term uncommitted vs. long-term committed) that a woman is pursuing (Provost et al., 2006; Waynforth et al., 2005; although see Glassenberg, Feinberg, et al., 2010), as indexed by SOI-R score.

\section{General discussion}

Across two experiments, we have shown that women who tend to favor short-term, uncommitted relationships show

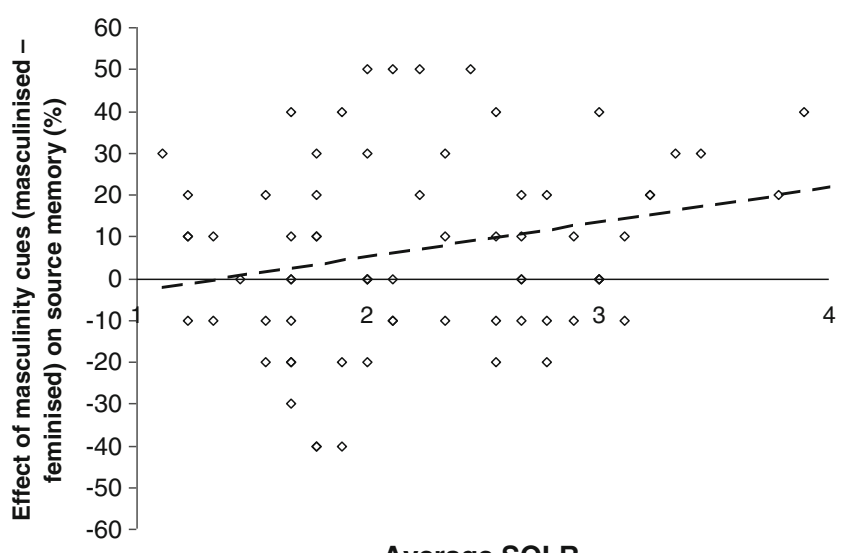

\section{Average SOI-R}

Fig. 3 Experiment 2 data showing the relationship between average SOI-R score and the effect of masculinizing men's faces (axes as per Fig. 2). Higher average SOI-R scores are once again associated with better source memory for masculinized versus feminized faces, while lower average SOI-R scores are associated with better source memory for feminized versus masculinized faces, $r(71)=.25, p<.05$ enhanced ability to recollect a quite arbitrary sourcespecifying detail (the color of the frame surrounding a facial image at encoding) that is triggered by cues of enhanced facial masculinity. Conversely, to the extent that women appear to favor an alternative strategy involving long-term, committed relationships, recollection is enhanced for feminized versus masculinized facial images. These findings replicate preference-linked patterns of bias in women's object recognition memory obtained in three prior experiments (Allan et al., 2012a; see also Smith et al., 2012), and in conjunction with the two studies reported here, we would suggest that strong evidence now exists for the conclusion that cues of masculinity trigger a systematic, possibly functional, response within women's episodic memory. This response acts to enhance the retention of otherwise quite arbitrary episodic details associated with men who happen to display desirable sexually dimorphic characteristics.

A key point to bear in mind is that exaggerated and reduced cues of male sexual dimorphism trigger opposite effects on women's memory, according to the kind of relationship that a woman prefers to pursue. Our data therefore show that bias in the ability to retain episodic details from encounters with men may be influenced by the significance of their sexually dimorphic characteristics as defined by a women's mate choice strategy. To our knowledge, this is the first direct evidence of a link between memory biases and adaptive mate choice strategies in women. We conclude that our findings suggest the existence of a mating mode in women's memory that is triggered by desirable sexually dimorphic cues. But before discussing the implications of our findings, it is worth pointing out some limitations of our present approach, which involved online data collection and explicitly capitalizing upon systematic individual differences in mating strategy.

As was just noted, because the direction of the memory bias reverses across different individuals, at the group level in Experiment 1 this resulted in no net (i.e., main) effect of the manipulated sexual dimorphism variable. Furthermore, dichotomizing the SOI-R variable in order to obtain significant main effects within high versus low SOI-R subgroups was also unsuccessful. In addition, it is evident that group performance in both experiments was at or near floor, even in Experiment 2 when participants were only asked to encode 20 facial images. It may be desirable to overcome these limitations in future research, even though they did not prevent us from finding a robust and replicable predictive relationship between SOI and the effect of masculinity cues upon women's recollection. Nevertheless, one possibility would be to experimentally manipulate whether women evaluate men in terms of short- versus long-term relationships, which has been shown to have systematic effects at the group level on women's preferences for masculinized versus feminized facial traits in men (e.g., Little, Jones, 
Penton-Voak, Burt, \& Perrett, 2002). This approach may encourage stronger effects of sexual dimorphism upon memory to emerge at a group level, and it may also be a more suitable approach for lab-based work, one that would also allow measures to improve performance levels per se, at the group level.

Returning to the implications of our findings, we would suggest that a more complete understanding of adaptive mate choice in humans has to involve models that are cognitively elaborate, in the sense that they include a functional contribution from perceptual and mnemonic processes (see, e.g., Bateson \& Healy, 2005). Memory function, however, has been largely ignored within the mate choice literature, which has tended to focus upon perceptual processes involved in attractiveness judgments about the opposite sex. Hence, we hope that one contribution of our present findings may be to encourage researchers interested specifically in mate choice to explicitly consider proximal mechanisms that involve the different structural and representational components identified in cognitive research on memory - for example, working memory, long-term memory, and, in particular, the interplay between semantic and episodic knowledge (Klein, Cosmides, Tooby, \& Chance, 2002).

Conversely, cognitive research on memory from a functionalist perspective may benefit greatly by closer integration with the mate choice literature. Prior studies have, for example, examined memory for attractive versus unattractive members of the opposite sex but either did not incorporate factors that systematically modulate attractiveness judgments or have failed to find evidence of a link between such factors and the effect of men's attractiveness upon women's memory (Anderson et al., 2010; Becker et al., 2005; Maner et al., 2003). For example, Becker et al. examined whether women's ability to localize male and female faces, using a game of "concentration," or "Pelmanism," was modulated by the attractiveness of the faces. Using prerated attractive versus unattractive faces, Becker et al. found that their women participants had better spatial localization of attractive versus unattractive women and that attractive men were most poorly localized. In an earlier study, Maner et al. reported similar findings for facial recognition but, contrary to our results here, found no evidence of any relationship of socio-sexual orientation (using the original, unrevised SOI questionnaire) and the effect of male facial attractiveness on women's facial recognition ability. It is therefore possible that the present findings may be specific to women's memory for episodic details associated with desirable men and may not generalize to their memory for men's faces per se.

Becker et al.'s (2005) results indicate, however, that women's spatial localization ability may be enhanced for attractive versus unattractive women, and their ability to remember men's faces and their locations seems to be hindered by men's attractiveness. Following up on this result, Anderson et al. (2010) found a similar intrasexual bias in women's face recognition memory for other attractive versus unattractive women but no such effect for men's faces. Anderson et al. also reported that the intrasex bias was not itself modulated according to whether women were currently in a fertile (i.e., peri-ovulatory) or nonfertile phase of their menstrual cycle. These findings are highly intriguing, when considered alongside our own results here and in our prior studies (Allan et al., 2012b; Smith et al., 2012). It would appear that women may have an enhanced spatial memory for other attractive versus unattractive women that is functionally decoupled from their mating strategy, at least so far as this is indexed by such measures as their sociosexual orientation. In contrast, our findings indicate that women do have enhanced memory for apparently more arbitrary episodic details associated with strategically desirable men. Collectively, these disparate findings suggest that there could be a functional dissociation within women's memory for the different kinds of episodic content available from encounters with attractive women versus desirable men.

Anderson et al. (2010) have suggested that the failure to find biases in women's memory linked to attractive versus unattractive men may reflect the relative unimportance of men's attractiveness per se in the context of mate choice. They suggested that other characteristics, such as those that suggest social standing, dominance, or the ability to accrue resources, may need to be manipulated in order to make a man more memorable. To the extent that cues of sexual dimorphism convey such information (e.g., Fink et al., 2007; Jones, Main, DeBruine, Little, \& Welling, 2010; Mueller \& Mazur, 1996), our present findings lend support to their suggestion. There is, however, a substantial remaining issue concerning the functional argument for women's enhanced retention of apparently trivial details, albeit that these details are associated with men who display characteristics strategically desirable in a potential partner.

First, it is worth reiterating that in Experiment 1, we found that women's SOI-R score captured a systematic effect of masculinity cues within both the male and the female faces. We pointed out that this finding is not necessarily contrary to a mate choice interpretation of the memory bias, because it is feasible that sensitivity to cues of masculinity in other women's faces could be a functionless byproduct of sensitivity to such cues in men's faces. As was noted above in the discussion of Experiment 1's findings, it is unlikely that the effect in women's faces is simply due to attractiveness, because women typically rate masculinized women's faces as less attractive than feminized women's faces (e.g., Perrett et al., 1998; Rhodes et al., 2006). Moreover, on the basis of the prior studies from Maner, Kenrick, and colleagues, we would expect that attractiveness effects on women's memory should lead to enhanced 
recollection for the feminized versus masculinized women's faces, which is contrary to the overall pattern that we observed in Experiment 1. Hence, the modulating effect of masculinity cues in women's faces upon women's memory is, in our opinion, unlikely to reflect the perceived attractiveness of those faces. However, more work is needed to examine why preference-linked effects of facial masculinity cues on object recognition are specific to male faces (Allan et al., 2012a), whereas SOI-R effects of facial masculinity cues on source memory may be observed with male and female faces.

In a prior article (Allan et al., 2012b), we developed the initial arguments for a mating mode shaped by selection pressure acting upon women's ability to retain information from encounters with desirable men. We pointed out that this information may have considerable adaptive value if it improves women's ability to judge how specific men are likely to behave in particular future relationship contexts. This, in turn, may reduce the chances of a woman making a poor mate choice decision that could severely impact upon her reproductive fitness. Within this framework, we would suggest that there are two separable functional consequences of enhanced ability to recollect seemingly arbitrary episodic details. The first is that the future utility of any piece of information may be hard to predict and that it may, therefore, be functional to enhance the encoding of information associated with desirable men without regard to its apparent utility in the present moment. Within this account, we would further suggest that retention of such details could play a specific scope-limiting role, as suggested by Klein et al. (2002), setting bounds on general inferences about male behavior within relationships. These generalizations are essentially stereotypical judgments about male behavior, triggered by their cues of masculinity, which involve inferences about fidelity, parental investment, and related character dispositions (e.g., coldness or warmth) (e.g., Perrett et al., 1998). As was pointed out by Klein et al., such generalities may be functionally bounded with the aid of information gained from one's experiences with specific individuals. A key prediction of this account is that the stereotype relevance of information associated with desirable men may be an additional important factor to consider, and this is a prediction that we are currently examining.

By invoking the encoding specificity principle (described first by Thomson \& Tulving, 1973), further functional consequences may follow from enhanced memory for episodic details from an encounter with a desirable man. Even the most trivial of episodic detail encoded during an encounter with a desirable man may act as an effective cue for the reinstatement of other elements of that episode, including elements that could have more apparent significance. Hence, by this argument, enhanced retention of such "peripheral" or innocuous details may, in the future, facilitate recollection of other pieces of information that could be significant in and of themselves. Hence, the enhanced ability to recollect an apparently insignificant detail is not functional because the detail is itself relevant to mate choice but may occur, instead, because future encounters with that detail may act as a more effective retrieval cue for the encounter itself, possibly including information that does have more apparent significance. This argument appears to predict that women's face recognition memory may be enhanced for men who display desirable sexually dimorphic cues, a prediction that we have not so far tested. If this prediction is proven correct, it would reinforce the point made by Anderson et al. (2010) noted above, which is that their failure to find biases in women's memory linked to attractive versus unattractive men may reflect the relative unimportance of men's attractiveness per se in the context of mate choice. We also suggest that this point may be important to bear in mind from the functionalist perspective, generally speaking, when considering which particular kinds of episodic information it may or may not be important to remember a priori within any evolutionarily important domain, and not just within mate choice (an issue tackled, for example, by Nairne et al., 2012).

A final point is that although we have framed our paper in explicitly functionalist terms, we have previously suggested that the goal-based model of episodic memory provided by Conway (2009) might provide an alternative account (Allan et al., 2012a). This model proposes that the relevance of episodic details to one's currently active goals can modulate how these details are encoded and retrieved. Our present findings fit very well with this idea and provide some measure of support for Conway and colleagues' model. That is, individuals with high versus low SOI-R scores may have functional biases within their episodic memory because they hold different underlying mating goals - that is, to obtain a partner with exaggerated versus reduced sexually dimorphic characteristics, respectively. A point worth noting, however, is that goal relevance is not operating simply as a factor that enhances attention to some information at the expense of less relevant information. Were this the case, we might expect to find that mere preference for or attraction to a class of face should trigger a corresponding enhancement for associated details in memory. Instead, as was noted above, the available data already suggest that preference is not sufficient to trigger the memory bias. For example, we observed (Allan et al., 2012b) that women significantly preferred feminized versus masculinized women's faces, but this did not lead to enhanced recognition of objects paired with feminized women's faces. Similarly, we also observed (Allan et al., 2012a) that men significantly preferred feminized to masculinized women's faces, but this preference did not trigger enhanced memory for objects paired with feminized women's faces. Hence, the available data suggest that the enhancing effects of men's 
facial (and vocal) masculinity upon women's episodic memory are not a simple by-product of enhanced attention to preferred, attractive stimuli.

In conclusion, our article has examined whether bias in women's memory for episodic details associated with desirable men (Allan et al., 2012b; see also Smith et al., 2012) can be linked to adaptive variation in mate choice strategies across individuals. Our findings, across two experiments, indicate that this is the case and, thereby, provide substantial support for the emerging functionalist perspective on memory. We suggest that our findings reveal the existence of a mode within episodic memory that enhances retention of information that could impact upon women's future reproductive success by aiding their ability to balance the pros and cons of mating with particular masculine or feminine men.

\section{References}

Allan, K., Jones, B. C., DeBruine, L. M., \& Smith, D. (2012a). Evidence of adaptation for mate choice within women's memory. Evolution and Human Behavior, 33, 193-199.

Allan, K., Midjord, J. P., Martin, D., \& Gabbert, F. (2012b). Memory conformity and the perceived accuracy of self versus other. Memory and Cognition, 40, 280-286.

Anderson, U. S., Perea, E. F., Becker, D. V., Ackerman, J. M., Shapiro, J. R., Neuberg, S. L., et al. (2010). I only have eyes for you: Ovulation redirects attention (but not memory) to attractive men. Journal of Experimental Social Psychology, 46, 804-808.

Andrews, P. W., Gangestad, S. W., \& Mathews, D. (2002). Adaptationism - how to carry out an exaptationist program. The Behavioral and Brain Sciences, 25, 489-553.

Bateson, M., \& Healy, S. D. (2005). Comparative evaluation and its implications for mate choice. Trends in Ecology \& Evolution, 20, 659-664.

Becker, V. D., Kenrick, D. T., Guerin, S., \& Maner, J. K. (2005). Concentrating on beauty: Sexual selection and socio-spatial memory. Personality and Social Psychology Bulletin, 31, 1643-1652.

Bell, R., Buchner, A., Kroneisen, M., \& Giang. (2012). On the flexibility of social source memory: A test of the emotional incongruity hypothesis. Journal of Experimental Psychology: Learning, Memory, and Cognition, 38, 1512-1529.

Boothroyd, L. G., Jones, B. C., Burt, D. M., DeBruine, L. M., \& Perrett, D. I. (2008). Facial correlates of sociosexuality. Evolution and Human Behavior, 29(3), 211-218.

Burns, D. J., Burns, S. A., \& Hwang, A. J. (2011). Adaptive memory: Determining the proximate mechanisms responsible for the memorial advantages of survival processing. Journal of Experimental Psychology: Learning, Memory, and Cognition, 37, 206-218.

Buss, D. M., Haselton, M. G., Shackelford, T. K., Bleske, A. L., \& Wakefield, J. C. (1998). Adaptations, exaptations, and spandrels. American Psychologist, 53, 533-548.

Conway, M. A. (2009). Episodic memories. Neuropsychologia, 47, 2305-2313.

DeBruine, L. M., Jones, B. C., Little, A. C., Boothroyd, L. G., Perrett, D. I., Penton-Voak, I. S., et al. (2006). Correlated preferences for facial masculinity and ideal or actual partner's masculinity. Proceedings of the Royal Society of London B, Biological Sciences, 273, 1355-1360.
Fink, B., Neave, N., \& Seydel, H. (2007). Male facial appearance signals physical strength to women. American Journal of Human Biology, 19, 82-87.

Fink, B., \& Penton-Voak, I. S. (2002). Evolutionary psychology of facial attractiveness. Current Directions in Psychological Science, $11,154-158$.

Gangestad, S. W., \& Simpson, J. A. (2000). The evolution of human mating: Trade-offs and strategic pluralism. The Behavioral and Brain Sciences, 32, 573-644.

Gangestad, S. W., Thornhill, R., \& Garver-Apgar, C. E. (2010). Men's facial masculinity predicts changes in their female partners' sexual interests across the ovulatory cycle, whereas men's intelligence does not. Evolution and Human Behavior, 31, 412-424.

Glassenberg, A. N., Feinberg, D. R., Jones, B. C., Little, A. C., \& DeBruine, L. M. (2010). Sex-dimorphic face shape preference in heterosexual and homosexual men and women. Archives of Sexual Behavior, 39, 1289-1296.

Hughes, S. M., Dispenza, F., \& Gallup, G. G., Jr. (2004). Ratings of voice attractiveness predict sexual behavior and body configuration. Evolution and Human Behavior, 25, 295-304.

Jaeger, A., Lauris, P., Selmeczy, D., \& Dobbins, I. G. (2012). The cost and benefits of memory conformity. Memory \& Cognition, 40(1), 101-112.

Johnson, M. K., Hashtroudi, S., \& Lindsay, D. S. (1993). Source monitoring. Psychological Bulletin, 114, 3-28.

Jones, B. C., DeBruine, L. M., Little, A. C., Conway, C. A., Welling, L. L. M., \& Smith, F. G. (2007). Sensation seeking and men's face preferences. Evolution and Human Behavior, 28, 439-446.

Jones, B. C., Main, J. C., DeBruine, L. M., Little, A. C., \& Welling, L. L. M. (2010). Reading the look of love: Sexually dimorphic cues in opposite-sex faces influence gaze-categorization. Psychological Science, 21, 796-798.

Justin, H. (2007). Distinguishing byproducts from non-adaptive effects of algorithmic adaptations. Evolutionary Psychology, 5(1), 47-51.

Klein, S. B., Cosmides, L., Tooby, J., \& Chance, S. (2002). Decisions and the evolution of memory: Multiple systems, multiple functions. Psychological Review, 109, 306-329.

Landolt, M. A., Lalumiere, M. L., \& Quinsey, V. L. (1995). Sex differences in intra-sex variations in human mating tactics: An evolutionary approach. Ethology and Sociobiology, 16, 3-23.

Little, A. C., Jones, B. C., \& DeBruine, L. M. (2011). Facial attractiveness: Evolutionary based research. Philosophical Transactions of the Royal Society B, Biological Sciences, 366, 1638-1659.

Little, A. C., Jones, B. C., Penton-Voak, I. S., Burt, D. M., \& Perrett, D. I. (2002). Partnership status and the temporal context of relationships influence human female preferences for sexual dimorphism in male face shape. Proceedings of the Royal Society of London, 269, 1095-1100.

Little, A. C., Jones, B. C., Waitt, C., Tiddeman, B. P., Feinberg, D. R., \& Perrett, D. I. (2008). Symmetry is related to sexual dimorphism in faces: Data across culture and species. PLoS One, 3, e2106.

MacCallum, R. C., Zhang, S., Preacher, K. J., \& Rucker, D. D. (2007). On the practice of dichotomization of quantitative variables. Psychological Methods, 7, 19-40.

Maner, J. K., Kenrick, D. T., Becker, D. V., Delton, A. W., Hofer, B., Wilbur, C. J., et al. (2003). Sexually selective cognition: Beauty captures the mind of the beholder. Journal of Personality and Social Psychology, 85(6), 1107-1120.

Mueller, U., \& Mazur, A. (1996). Facial dominance in west point cadets predicts military rank $20+$ years later. Social Forces, 74 (3), 823-850.

Nairne, J. S., Pandeirada, J. N. S., \& Thompson, S. R. (2008). Adaptive memory: The comparative value of survival processing. Psychological Science, 19, 176-180.

Nairne, J. S., Thompson, S. R., \& Pandeirada, J. N. S. (2007). Adaptive memory: Survival processing enhances retention. Journal of 
Experimental Psychology: Learning, Memory, and Cognition, 33, 263-273.

Nairne, J. S., VanArsdall, J. E., Pandeirada, J. N. S., \& Blunt, J. R. (2012). Adaptive memory: Enhanced location memory after survival processing. Journal of Experimental Psychology: Learning, Memory, and Cognition, 38(2), 495-501.

New, J., Krasnow, M. M., Truxaw, D., \& Gaulin, S. J. C. (2007). Spatial adaptations for plant foraging: Women excel and calories count. Proceedings of the Royal Society B, Biological Sciences, 274, 2679-2684.

Penke, L., \& Asendorpf, J. B. (2008a). Beyond global sociosexual orientations: A more differentiated look at sociosexuality and its effects on courtship and romantic relationships. Journal of Personality and Social Psychology, 95, 1113-1135.

Penke, L., \& Asendorpf, J. B. (2008b). The revised sociosexual orientation inventory (SOI-R). Retrieved November 19, 2012, from http://www.larspenke.eu/soi-r/index.html

Penton-Voak, I. S. (2011). In retreat from nature? Successes and concerns in Darwinian approaches to facial attractiveness. Journal of Evolutionary Psychology, 9, 173-193.

Perrett, D. I., Lee, K. J., Penton-Voak, I., Rowland, D., Yoshikawa, S., Burt, D. M., et al. (1998). Effects of sexual dimorphism on facial attractiveness. Nature, 394, 884-887.

Price, M. E., Kang, J., Dunn, J., \& Hopkins, S. (2011). Muscularity and attractiveness as predictors of human egalitarianism. Personality and Individual Differences, 50, 636-640.

Provost, M. P., Kormos, C., Kosakoski, G., \& Quinsey, V. L. (2006). Sociosexuality in women and preference for facial masculinization and somatotype in men. Archives of Sexual Behavior, 35, 305-312.

Rantala M. J, Moore F. R., Skrinda I., Krama T,. Kivleniece I., Kecko S., \& Krams I. (2012). Evidence for the stress-linked immunocompetence handicap hypothesis in humans. Nature Communication, 3, article 694

Rehnman, J., \& Herlitz, A. (2007). Women remember more faces than men do. Acta Psychologica, 124, 344-355.

Rhodes, G., Chan, J., Zebrowitz, L. A., \& Simmons, L. W. (2006). Does sexual dimorphism in human faces signal health? Proceedings of the Royal Society B, Biological Sciences, (supp) 270, 93-95.

Sell, A., Cosmides, L., Tooby, J., Sznycer, D., von Rueden, C., \& Gurben, M. (2009). Human adaptations for the visual assessment of strength and fighting ability from the body and face. Proceedings of the Royal Society of London B, Biological Sciences, 276, 575-584.

Sherry, D. F., \& Schacter, D. L. (1987). The evolution of multiple memory systems. Psychological Review, 94, 439-454.

Smith, D. S., Jones, B. C., Feinberg, D. R., \& Allan, K. (2012). A modulatory effect of male voice pitch on long-term memory in women: Evidence of adaptation for mate choice? Memory \& Cognition, 40, 135-144.

Thomson, D \& Tulving, E (1973). Encoding specificity and retrieval processes in episodic memory. Psychological Review, $80,352-373$.

Thornhill, R., \& Gangestad, S. W. (2006). Facial sexual dimorphism, developmental stability, and susceptibility to disease in men and women. Evolution and Human Behavior, 27, 131-144.

Tiddeman, B. P., Perrett, D. I., \& Burt, D. M. (2001). Prototyping and transforming facial textures for perception research. IEEE Computer Graphics and Applications, 21, 42-50.

Waynforth, D., Delwadia, S., \& Camm, M. (2005). The influence of women's mating strategies on preference for masculine facial architecture. Evolution and Human Behavior, 26, 409-416. 